JEFAS

23,44

4

Received 3 June 2017 Revised 16 August 2017 Accepted 18 August 2017

\section{Determinantes y pronóstico de la actividad bursátil del mercado accionario colombiano}

\author{
David Agudelo, Diego A. Agudelo y Julián Peláez \\ Department of Finance, Universidad EAFIT, Medellin, Colombia
}

\section{Resumen}

Propósito - Se estudian los determinantes y la evolución de la actividad bursátil mensual en el mercado accionario colombiano de 2007 a 2016.

Diseño/metodología/enfoque - Para ello se emplean modelos de series de tiempo tipo ARIMAX y GARCH, incluyendo variables exógenas, recomendadas por la literatura previa.

Hallazgos - Encontramos que la actividad bursátil puede ser pronosticada en buena parte por el valor rezagado a un mes y las innovaciones de cinco y 12 meses. También contribuyen a predecirla, como variables exógenas, una dummy de rendimientos positivos en los últimos tres meses, la presencia de emisiones primarias y el índice VIX de volatilidad del SP500. Estos resultados se mantienen en un alto grado al emplear medidas alternativas de actividad bursátil, el número total de operaciones y la rotación.

Implicaciones prácticas - Se propone un modelo de predicción de la actividad bursátil que puede servir de modelo para otros mercados accionarios de Latinoamérica. El modelo obtenido es altamente predictivo del valor transado total del mercado al siguiente mes. La estimación de la actividad bursátil es de utilidad para instituciones como la Bolsa de Valores de Colombia, reguladores de los mercados financieros, así como para grandes inversionistas institucionales.

Implicaciones sociales - El propósito central de los mercados financieros secundarios consiste en facilitar la transacción de activos financieros, lo que debe reflejarse en alta actividad bursátil, tanto en número de operaciones como en valor transado total. La posibilidad de transar altos montos es una medida importante del desarrollo de un mercado financiero. De esta manera, el modelo aquí propuesto puede usarse para monitorizar y explicar el desarrollo del mercado. En particular, se evidencia el nocivo efecto de la debacle de Interbolsa a finales de $2012 \mathrm{y}$ el positivo efecto de las emisiones primarias.

Originalidad/valor - Este es el primer paper en estudiar la actividad bursátil del mercado accionario colombiano en años recientes. Sirve como modelo para el estudio y seguimiento de esta variable en otros mercados accionarios latinoamericanos.

Palabras clave - Actividad bursátil, Mercados accionarios, Modelos ARMA y GARCH, Valor transado

Tipo de artículo - Artículo de investigación

(C) David Agudelo, Diego A. Agudelo and Julián Peláez. Published in Journal of Economics, Finance and Administrative Science. Published by Emerald Publishing Limited. This article is published under the Creative Commons Attribution (CC BY 4.0) licence. Anyone may reproduce, distribute, translate and create derivative works of this article (for both commercial and non-commercial purposes), subject to full attribution to the original publication and authors. The full terms of this licence may be seen at http://creativecommons.org/licenses/by/4.0/legalcode

Esta investigación es resultado del proyecto de grado de David Agudelo y Julián Peláez, con la asesoría de Diego A. Agudelo en la Maestría en Administración Financiera de la Universidad EAFIT. Agradecemos a la Bolsa de Valores de Colombia, en particular a Adriana Cárdenas, por suministrar la base de datos. 


\begin{abstract}
Purpose - To study the determinants and evolution of the trading activity in the Colombian Stock Market from 2007 to 2016 .
\end{abstract}

Design/methodology/approach - ARMA time series models were used, including several explanatory variables recommended by previous literature.

Findings - We find that stock market activity can be predicted to a large extent by its lags, and that positive returns in the last three months, emissions and the VIX index are also explicative variables, as suggested by empirical studies in other countries and theoretical models of market microstructure. These results are robust by using alternative measures of trading activity, total number of trades and turnover.

Originality/value - The main contribution of this study is the analysis of the trading activity of the Colombian Stock Market, a critical variable for monitoring the development of any financial market.

Keywords Stock market activity, Trading value, Stock market, ARMA models

Paper type Research paper

\section{Introducción}

La actividad bursátil, medida como volumen, valor transado o número de operaciones, es el propósito central de los mercados financieros secundarios. Naturalmente, su variabilidad y reducción sustancial afecta las decisiones de los agentes, en particular de aquellos que gestionan portafolios de gran tamaño, tanto para sus posiciones activas como pasivas. Asimismo, se ha identificado como una variable importante del desarrollo de un mercado bursátil. Por esto, es importante comprender las variables que la determinan y su evolución, especialmente en un mercado accionario emergente como el colombiano.

Es importante comenzar aclarando que la actividad bursátil y la liquidez son conceptos relacionados, mas no equivalentes[1]. Harris (2003), define la liquidez como la facilidad de transar un activo a un bajo costo, en grandes volúmenes y en corto tiempo. Usualmente, la liquidez es estimada mediante medidas de costos de transacción, como el margen entre la oferta y la demanda y el impacto en el precio. Por otro lado, la actividad bursátil es medida como la rotación, valor transado, número de operaciones, entre otros. El hecho de que un activo sea transado frecuentemente no lo hace necesariamente poco costoso de transar (líquido) ni viceversa. Este estudio se centrará en la actividad bursátil para el mercado accionario colombiano y no se ocupará de la liquidez.

Tres principales interrogantes enmarcan el presente trabajo investigativo: ¿cuál ha sido la evolución de la actividad bursátil del mercado accionario en la Bolsa de Valores de Colombia (Bvc) entre 2007 y 2016? ¿Qué variables la explican? ¿Qué eventos han impactado su comportamiento? Para ello, se emplea información de transacciones diarias por tipo de agente de la BVc, entre enero de 2007 y diciembre de 2016. Además, se plantea un modelo de pronóstico del comportamiento de la actividad bursátil, que incluye variables explicativas buscando predecir con razonable certeza la actividad bursátil del siguiente mes. Así, este estudio pretende contribuir a la literatura y al desarrollo del mercado accionario colombiano, con información relevante para una mejor comprensión de la actividad bursátil, su comportamiento en el tiempo y las variables que la determinan.

Consideramos que el principal aporte de este estudio es el análisis de la variable de actividad bursátil del mercado accionario colombiano, medida como valor transado, número de operaciones y rotación, de importancia central para monitorear el desarrollo de un mercado de activos financieros. Destacamos los dos siguientes trabajos como los más relacionados al presente estudio. En primer lugar, el de Griffin et al. (2006), que estudia un grupo de 46 mercados desarrollados y emergentes, y encuentran que la volatilidad y los rendimientos semanales pasados tienen relación positiva con la actividad bursátil. En
Determinantes y pronóstico de la actividad bursátil 
JEFAS 23,44

segundo lugar, Agudelo (2009) que estudia el mercado accionario de la BVC en un período previo (1997-2007). Empleando modelos de series de tiempo, el autor pone de presente que la actividad bursátil depende de los rendimientos contemporáneos y sus rezagos, disminuyendo antes de los anuncios macroeconómicos. A diferencia de este último, en el presente se estudian los determinantes de actividad bursátil y se plantea un modelo econométrico de predicción de la misma[2].

Estos resultados son de potencial interés para tres tipos de instituciones: en primer lugar, para inversionistas institucionales con altas posiciones en acciones como los fondos de pensiones y las carteras colectivas. En segundo lugar, para aquellos responsables de un volumen importante de transacciones como las sociedades comisionistas de Bolsa (en adelante SCB) y los Family offices. Estos dos tipos de inversionistas institucionales pueden emplear el modelo que aquí se presenta para encontrar los meses más adecuados en el desarrollo de sus estrategias activas, ya que una mayor actividad bursátil está asociada a mercados más líquidos y de mayor profundidad (Bayraktar, 2014). En tercer lugar, las instituciones cuyos ingresos dependen de la actividad bursátil misma, como la Bolsa de Valores de Colombia y las SCB. Por ejemplo, una SCB cuyos ingresos dependen en buena parte de las comisiones por la compra y venta de títulos valores para terceros, está interesada en pronosticar la actividad bursátil y con ello realizar sus estimaciones presupuestales. Por su parte, para la BVC es importante hacerle seguimiento y estimar a futuro la actividad bursátil, no solo para proyectar mejor sus ingresos por tarifas, sino también para monitorear el desarrollo del mercado.

Para abordar la situación de estudio, el resto de este artículo está organizado de la siguiente manera: primero, se realiza un análisis detallado del comportamiento de la actividad bursátil en el caso colombiano y su evolución en el tiempo. Inicialmente, se discuten los acontecimientos potencialmente más relevantes para los valores transados del mercado accionario entre 2007 a 2016. A continuación, en la revisión de la literatura, se referencian diversos estudios que explican el comportamiento de la actividad bursátil e identifican sus variables determinantes.

En el apartado de metodología se presentan y discuten las posibles variables explicativas basadas en la literatura, y se plantea un proceso estocástico que tiene capacidad predictiva mediante la modelación econométrica de series temporales, en el contexto del análisis ARIMA de Box et al. (1994). Se encuentra que las emisiones, la persistencia de los rendimientos pasados positivos, la volatilidad de los mercados internacionales y la actividad bursátil rezagada son determinantes estadísticamente significativos de la actividad bursátil en un mes dado. También se comprueba la robustez de los hallazgos empleando dos medidas alternativas de actividad bursátil: el número de operaciones y la rotación. Se ejemplifica la potencial utilidad de los resultados del modelo para los diferentes agentes del mercado. Finalmente, se presentan las conclusiones del estudio.

\section{Actividad bursátil en Colombia, 2007-2016}

La Bolsa de Valores de Colombia (BVC) es un mercado bursátil emergente y de tamaño reducido[3], como se aprecia en la Figura 1, que compara el número de emisores en la BVc con respecto al promedio de emisores de las Bolsas por región[4]. Más aún, solo entre 10-25 compañías de las 70 listadas en BVC se transan con suficiente frecuencia[5]. Sin embargo, la actividad bursátil del mercado accionario colombiano ha evolucionado de manera importante en los últimos diez años, período del que se ocupa el presente estudio, como se ilustra en la Figura 2. Destacamos los siguientes hitos en la evolución del mercado de acciones en la BVC: 


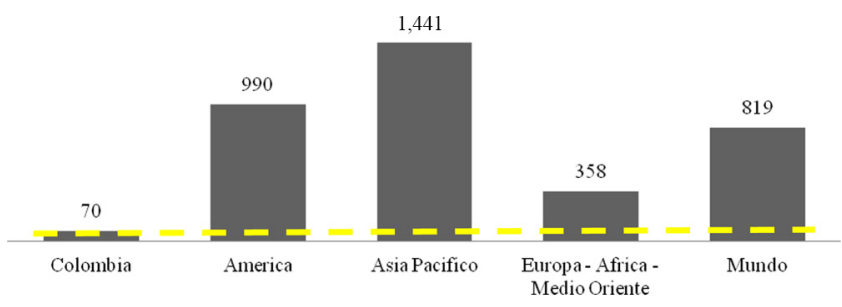

Notas: Se compara el número de emisores promedio de las Bolsas de Valores en cada región. Datos de World Federation Exchanges al cierre del 31 de diciembre de 2016. Elaboración propia

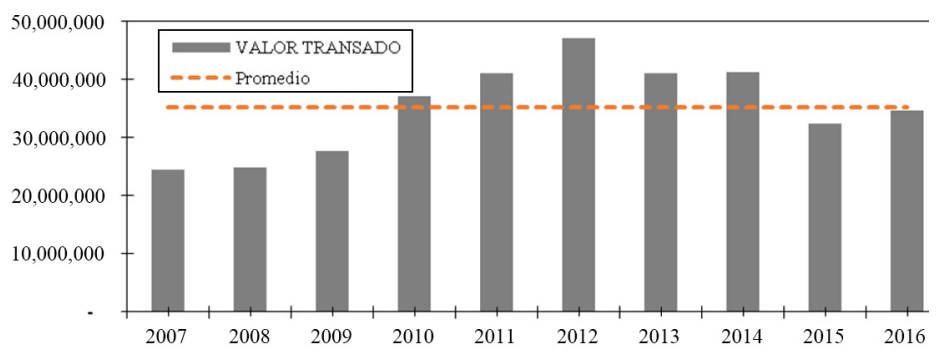

Notas: Se ilustra el comportamiento del valor transado anual en millones de pesos colombianos en la BVC, durante el período 2007-2016. Datos en millones de pesos. Elaboración propia
Determinantes y pronóstico de la actividad bursátil

Figura 1.

Número de emisores promedio por región
Figura 2.

Evolución de la actividad bursátil en Colombia

- Año 2007. Ecopetrol (la mayor empresa de Colombia) emite alrededor de 5.7 billones de pesos, en el mes de noviembre. Esta emisión propicia que más de 500 mil personas ingresen al mercado de valores por primera vez[6].

- Año 2009. En febrero se moderniza la operación del mercado accionario con la entrada en operación de la plataforma transaccional de la BVC X-Stream, que representó grandes adelantos en la rapidez, eficiencia y actividad bursátil de las transacciones en acciones (Agudelo et al., 2014).

- Año 2010. Crecimiento récord de la actividad bursátil, un 34.2 per cent con respecto al año 2009. Ha sido asociado a mayores ingresos de capitales globales hacia economías emergentes, en el contexto de la crisis de la zona Euro y de cambios regulativos en Colombia[7].

- Año 2011. Récord en emisiones primarias, ocho compañías que vendieron títulos por el valor de $\$ 12,9$ billones de pesos[8]. Para este año el valor transado aumentó un 10 con respecto al 2010[9].

- Año 2012. Aunque se registró un crecimiento de la actividad bursátil, el panorama del mercado accionario se vio opacado al final del año por la debacle de la mayor $\mathrm{SCB}$, "Interbolsa". En noviembre fue intervenida y liquidada por el ente regulador, la Superintendencia Financiera[10].

- Año 2013. La pérdida de confianza en el mercado accionario tras la debacle de Interbolsa permite explicar la importante disminución del 12.9 per cent de la 
JEFAS

23,44

8

Figura 3.

Actividad bursátil por tipo de inversionista
Figura 4.

Volatilidad de los mercados locales y extranjeros actividad bursátil en 2013 respecto de 2012, equivalente a seis billones de pesos (ver la Figura 3).

- Año 2015. La reducción adicional en 2015, que se muestra en la Figura 3, se asocia a la coyuntura económica global, que repercute de manera negativa en los mercados emergentes en general y en la BVC en particular. Los factores más determinantes fueron una importante disminución del precio del petróleo (35 per cent en 12 meses a partir de 29 de enero de 2015) y otros commodities, la devaluación del peso colombiano frente al dólar y el aumento de la volatilidad de los mercados financieros globales (ver la Figura 4). En consecuencia, la actividad bursátil en las acciones de la BVC se redujo un 21 per cent en 2015 con relación al anterior[11].

- Año 2016. Persisten niveles altos en la volatilidad de los mercados internacionales, esta vez asociados al Brexit (salida del Reino Unido de la Unión Europea) y a los resultados electorales en Estados Unidos. A su vez, en Colombia, el referendo de paz y la reforma fiscal, influenciaron la volatilidad del mercado accionario local. Sin

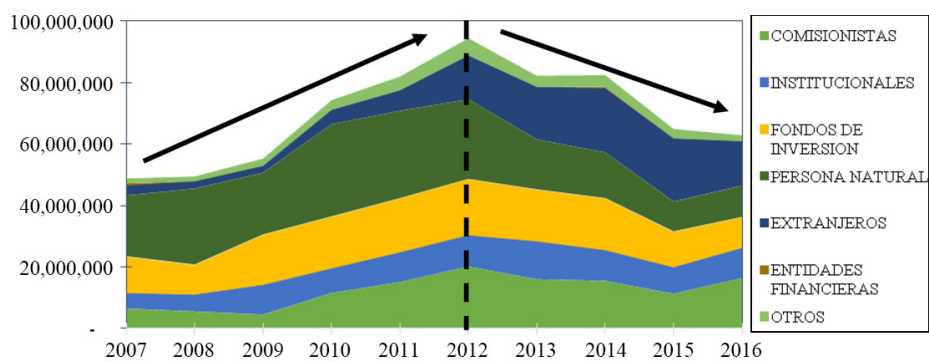

Notas: Se presenta la evolución del valor transado total (compras más ventas) en millones de pesos colombianos por tipo de inversionistas en la BVC desde enero de 2007 hasta noviembre de 2016. Datos en millones de pesos. Elaboración propia

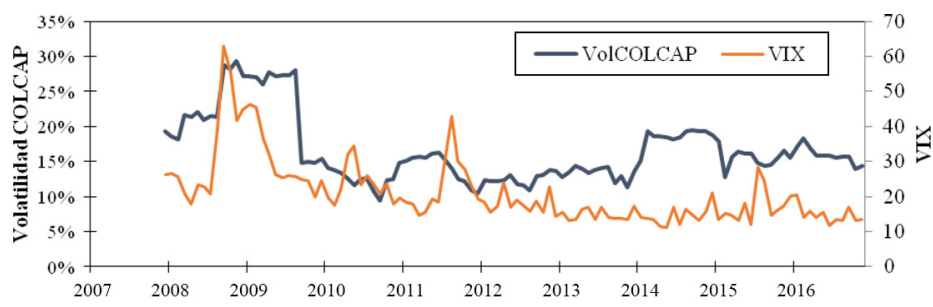

Notas: Se grafica el comportamiento del índice VIX (Chicago Board Options Exchange Market Volatility Index), expresada como volatilidad anual, y la volatilidad del índice COLCAP (calculada como la desviación estándar de los rendimientos mensuales, 12 meses corridos), mensual desde 2007 hasta 2016

Fuente: Bloomberg 
embargo, parte de la incertidumbre se resolvió con la firma del acuerdo de paz con las FARc (Fuerzas Armadas Revolucionarias de Colombia) y la aprobación de la reforma fiscal en el último trimestre del año. El índice colcAP se valorizó un 17.2 per cent en el año 2016, asociado al mejor desempeño de los precios de los commodities y la estabilidad en la tasa de cambio. Todo lo anterior refleja un aumento del valor transado del 6 per cent en 2016 con respecto a 2015[12].

\section{Determinantes y pronóstico de la actividad bursátil}

\section{Antecedentes}

La literatura seminal de microestructura de mercado (Kyle, 1985; Glosten y Milgrom, 1985) modela la actividad bursátil y la formación de precios de los activos financieros como producto de la interacción entre tres tipos de agentes: a) el creador de mercado, permanentemente dispuesto a comprar y vender el activo, b) agentes no informados (también llamados noise traders), que transan por choques exógenos de liquidez o por motivos infundados, sin basarse en información real, y c) agentes informados, que compran (venden) el activo con la certeza de que su precio actual es, al menos en expectativa, inferior (superior) al que tendrá cuando se publique información que ellos conocen o estiman con cierta certeza. Si bien dichos estudios buscan modelar los determinantes de la liquidez de los activos financieros, también tienen implicaciones para el comportamiento de la actividad bursátil. Concretamente, de estos modelos se infiere que la actividad bursátil aumenta con anticipación a períodos de mayor información (por ejemplo, reportes financieros, noticias macroeconómicas, anticipación a emisiones o adquisiciones), donde se espera una mayor actividad de los agentes informados. Asimismo, en cuanto a los agentes no informados, la actividad bursátil se verá afectada por choques de liquidez en la economía que los lleven a transar o episodios que dirijan su atención hacia los mercados bursátiles (por ejemplo, rendimientos pasados positivos, emisiones, crisis, escándalos bursátiles).

Diferentes autores se han ocupado del estudio empírico de variables de mercado que expliquen la variabilidad en la actividad bursátil. Por ejemplo, Gallant et al. (1992) encontraron que el volumen transado está positivamente correlacionado con los rendimientos y la volatilidad del mercado[13]. Chordia et al. (2001), confirman la relación positiva de los rendimientos y su volatilidad con la liquidez y la actividad bursátil[14], reportando además una reducción de esta última en los días viernes. Además, incluyeron anuncios macroeconómicos (PIB, IPC y desempleo) sin significancia estadística en sus resultados y encontraron que las tasas de interés tienen efecto negativo en la actividad bursátil[15]. Posteriormente, Chordia et al. (2002), contribuyendo a su estudio anterior, determinaron además que el desbalance en las órdenes (diferencia entre compras activas y ventas activas) afecta la actividad bursátil[16], y está fuertemente correlacionado con los rendimientos pasados del S\&P500[17],[18].

Griffin et al. (2006), examinan los rendimientos semanales pasados y la rotación (turnover) [19], encontrando una correlación positiva entre ambos, mediante un modelo vaR que además incorpora la volatilidad. Esta relación implica una asimetría en la relación entre rendimientos pasados y actividad bursátil. Concretamente, períodos anteriores de rendimientos positivos altos están relacionados con mayor volumen transado futuro. Los autores concluyen que la relación positiva es mayor en mercados accionarios menos eficientes, con restricciones para ventas en corto, con menor presencia de creadores de liquidez, y cuando la economía es más riesgosa y poco correlacionada con otras economías[20].

La relación asimétrica entre rendimientos pasados y actividad bursátil de dicho estudio también puede relacionarse con el llamado efecto disposición (disposition effect) registrado por primera vez por Shefrin and Statman (1985). Este efecto puede definirse como una 
JEFAS 23,44

propensión a vender rápidamente posiciones ganadoras y a mantener posiciones vendedoras. En la literatura de Behavioral Finance este efecto ha sido explicado desde la aversión a las pérdidas (Shefrin, 2002). Claramente, si el efecto disposición es común en el conjunto de inversionistas de un mercado, podrá explicar que rendimientos altos suelan ser seguidos por una alta actividad bursátil en el futuro, y que rendimientos negativos pasados conlleven a una reducción de la misma.

Chordia et al. (2006) se enfocan en los determinantes de la variación de la actividad bursátil. Argumentan que la actividad bursátil depende en parte de la liquidez y de la información que tengan los agentes. Reportan que los inversionistas individuales tienden a aumentar sus posiciones sobre las acciones más atractivas, según el tamaño, la edad de la firma, la razón de valor en libros a precio de mercado (book-to-market ratio) y el precio. Para medir la actividad bursátil utilizan el desbalance de órdenes y el turnover con datos de corte transversal de diez portafolios. Presentan evidencia de que la actividad bursátil aumenta con el crecimiento de la industria a la que pertenece la acción, menores costos de transacción y un mayor e-trading de agentes individuales. Así mismo, encuentran que las acciones con mejores rendimientos pasados tienden a atraer inversionistas individuales[21].

Glaser and Weber (2009), se enfocaron en investigar la relación entre la actividad bursátil y rendimientos pasados en cuanto a inversionistas individuales. Argumentan que los altos rendimientos de una acción aumentan la confianza de los inversionistas y los incita a invertir más. Encuentran que los rendimientos pasados de los portafolios de cada inversionista están positivamente correlacionados con su actividad bursátil (medida tanto en volumen como en número de transacciones). Muestran que después de rendimientos altos en sus portafolios, los inversionistas aumentan su exposición al riesgo y reducen su diversificación; esto no pasa tras bajos rendimientos del mercado; ellos explican estos hallazgos como consistentes con sesgos de autoatribución y diferencias de opinión[22].

Posteriormente Chordia et al. (2011) estudian la evolución reciente de la actividad bursátil y su impacto sobre la eficiencia del mercado, resaltando el aporte a ambas medidas del algorithmic trading y los brokers online. Encuentran que el aumento de la actividad bursátil está asociado a un mayor número de pequeñas transacciones que representan una creciente proporción del volumen transado. Reportan, además, que los inversionistas institucionales más que los individuos son responsables de la mayor parte del volumen transado y esto ha conllevado a aumentos en la eficiencia de mercado (medida por el variance ratio). Además, concluyeron que una reducción en los costos de transacción genera grandes aumentos en la actividad bursátil y reportan una tendencia de reducción en la volatilidad intradiaria y de aumentos de la sensibilidad del turnover a los rendimientos pasados[23].

En el caso colombiano, Agudelo (2009) estudia la actividad bursátil entre 1997-2007 medida como rotación y valor transado y explora sus determinantes. Encuentra que esta depende en buena medida de los rendimientos contemporáneos y sus rezagos y disminuye antes de los anuncios macroeconómicos. En opinión del autor, esto contrasta con lo reportado en Estados Unidos y evidencia la poca sofisticación del mercado accionario colombiano. En respuesta a su pregunta central, presenta evidencia sobre aumentos en el número de operaciones para el mercado colombiano tras la fusión de las tres Bolsas (Bogotá, Medellín y Cali) en julio de 2001, tanto para el mercado como un todo como para un número importante de acciones individuales. Además, identifica efectos estacionales de esta variable dentro del año y la semana, siendo los lunes y los viernes los días con menor actividad bursátil, así como los días inmediatamente anteriores y posteriores a los festivos.

En otro estudio del mercado accionario colombiano, Agudelo et al. (2014) estudiaron el efecto de la implementación de $X$-Stream en febrero de 2009, que presentó una mejora en la rapidez, efectividad y versatilidad de la transacción de acciones frente a la plataforma 
anterior. Los autores entregan evidencia de una mejora sustancial de la liquidez del mercado en general y de la actividad bursátil, sobre todo para las acciones de mediana bursatilidad.

En resumen, la revisión de la literatura muestra que la actividad bursátil está determinada por variables de mercado y variables exógenas como los rendimientos pasados, la volatilidad de los mercados, anuncios macroeconómicos, política monetaria, cambios estructurales y el comportamiento de la propia actividad bursátil en períodos anteriores. Todas estas variables deben ser tenidas en cuenta en un modelo que pretenda pronosticar la actividad bursátil.

\section{Datos y metodología}

\subsection{Medidas de la actividad bursátil}

En el presente estudio se utiliza el valor transado como medida de actividad bursátil, es decir, el monto total transado en pesos colombianos durante un período determinado de tiempo (en el caso analizado, se toman datos agregados mensuales).

Ecuación 1. Valor transado

$$
\text { ValTransadoMdo } t=\sum_{i=1}^{N} \text { ValTransado }_{i t}
$$

donde ValTransado ${ }_{i t}$ es el valor transado para la acción $i$ durante el período $t$.

Se escoge el valor transado como una medida más apropiada para el mercado accionario en lugar del volumen (número de acciones negociadas), la medida más común en el mercado de Estados Unidos, por dos razones: en primer lugar, el muy amplio rango de precios en los que se negocian las acciones en la BVC[24]. Esto haría inconsecuente sumar o comparar números de acciones negociadas entre diferentes especies (Agudelo, 2014). Por ejemplo, si se agrega la negociación de un millón de acciones de Fabricato a $\$ 10$ cada una con la de un millón de acciones de Banco de Bogotá a $\$ 60,000$, serían dos millones de acciones, pero para la primera especie se trata solo de diez millones de pesos, mientras que para la segunda son 60,000 millones de pesos, 6,000 veces más. En contraste, la mayoría de las acciones en los mercados de Estados Unidos está en un rango entre 10 y 80 dólares, y se mantienen en el mismo nivel mediante división de acciones (splits).

En segundo lugar, las comisiones en Colombia se manejan como porcentaje del valor negociado, no como en Estados Unidos, con tarifas fijas en dinero, que varían en rangos en función del número de acciones[25]. Dado que una de las posibles aplicaciones de la estimación de la actividad bursátil es la estimación de ingresos para comisionistas, se hace más relevante estimar el valor transado en el caso colombiano.

Se consideran, además, otras dos medidas alternativas de actividad bursátil como apropiadas para el mercado accionario colombiano. En primer lugar, el número de operaciones, variable muy empleada en los estudios de liquidez, por ejemplo, Stoll (2000), y que tiene la ventaja de no ser dominada por grandes transacciones cruzadas entre institucionales. Por otro lado, la rotación (turnover) que ha sido empleada por Griffin et al. (2006) and Lo y Wang (2000). Comparado con el valor transado, la rotación tiene la ventaja de permitir comparaciones entre períodos de tiempo distantes por no estar afectada por las valorizaciones de las acciones. Sin embargo, no tiene una interpretación tan directa para los operadores del mercado y, por ello, es de menor interés para los objetivos de este estudio.

\subsection{Modelo econométrico}

El análisis de series temporales como las que se emplean en este estudio ha sido frecuentemente empleado en finanzas. Este análisis clásico fue desarrollado por Box y

Determinantes y pronóstico de la actividad bursátil 
JEFAS

23,44
Jenkins (1973) a partir de procesos estacionarios[26], y ampliado por Engel (1982) para determinar un patrón estadístico para la varianza. Dada la frecuente heterocedasticidad de las series financieras se emplea un modelo de volatilidad condicional autorregresiva (ARCH) [27], cuya especificación general es:

Ecuación 2. Especificación ARCH:

$$
y_{t}=\varepsilon_{t} \sigma_{t}
$$

$$
\operatorname{VAR}\left(\varepsilon_{t}\right)=\sigma_{t}^{2}=\omega+\sum_{i=1}^{q} \alpha_{i} y_{t-i}^{2}
$$

donde la ecuación de $y_{t}$ incluye los términos del ARCH, los términos del modelo ARMA, y las variables exógenas en $X_{t}$ así:

$$
y_{t}=X_{t} \beta+A R M A(p, q)+\varepsilon_{t} \sigma_{t}
$$

Considerando que el proceso generador de los datos sigue un proceso autorregresivo y de medias móviles, debido a que involucra un componente inercial, cuya especificación general es[28]:

Ecuación 3. Especificación ARMA (p, q):

$$
\begin{aligned}
y_{t}= & X_{t} \beta+\rho_{1}\left(y_{t-1}-X_{t-1} \beta\right)+\rho_{2}\left(y_{t-2}-X_{t-2} \beta\right)+\ldots+\rho_{p}\left(y_{t-p}-X_{t-p} \beta\right) \\
& +\theta_{1} \varepsilon_{t-1}+\theta_{2} \varepsilon_{t-2}+\ldots+\theta_{q} \varepsilon_{t-q}+\varepsilon_{t}
\end{aligned}
$$

donde $\rho_{p}$ es el parámetro de autocorrelación de orden $p, \theta_{q}$ es el parámetro de media móvil de orden $q . \varepsilon_{t} \sim$ i.id. $\mathrm{N}\left(0, \sigma^{2}\right)$, es decir, es una perturbación ruido blanco. $X_{t}$ es un conjunto de variables de control.

Se realizan, además, las pruebas usuales sobre los residuales del modelo, para asegurar que cumplen con los supuestos. En concreto, se valida que los residuales sean ruido blanco, es decir que los residuales sigan un proceso con media cero y varianza constante, donde no exista correlación entre sus valores a través del tiempo.

\subsection{Actividad bursátil por tipo de inversionista}

Es de esperarse que la actividad bursátil de cada tipo de inversionista tenga características diferentes, correspondiendo con su estilo, plazo y montos de inversión. En la Tabla I, se presenta una clasificación general de los tipos de inversionistas en el mercado accionario colombiano y sus características más relevantes.

Clasificando la actividad bursátil por tipo de inversionista, en la Figura 5 se ve que las personas naturales y los fondos de inversión han sido vendedores netos en el período de estudio, mientras que los inversionistas extranjeros y los institucionales han sido compradores netos.

En general, las personas naturales han tenido una participación importante en el mercado bursátil colombiano, como lo muestran las Figuras 3 y 5 , comprando o vendiendo títulos valores directamente, en vez de hacerlo a través de inversionistas institucionales, 10 que es característico de un mercado emergente. Por ejemplo, Allen y Santomero (1998), presentan que en mercados accionarios desarrollados con costos de transacción bajos y facilidad en el acceso a la información, los intermediarios financieros tienen la mayor 


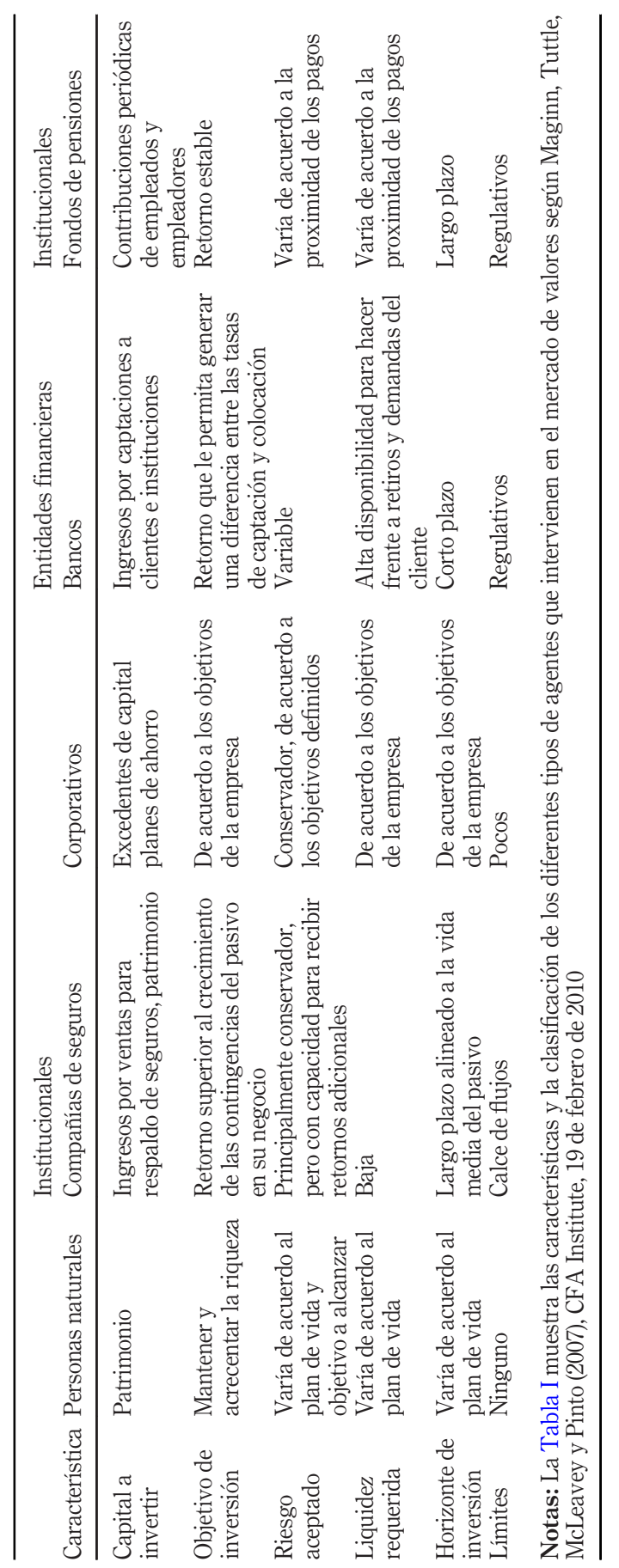

Determinantes y pronóstico de la actividad bursátil

Tabla I. Caracterización de algunos de los tipos de inversionistas 


\section{JEFAS 23,44}

\section{4}

\section{Figura 5.}

Compras vs ventas por tipo de inversionista
Tabla II.

Estadística descriptiva del valor transado por inversionista

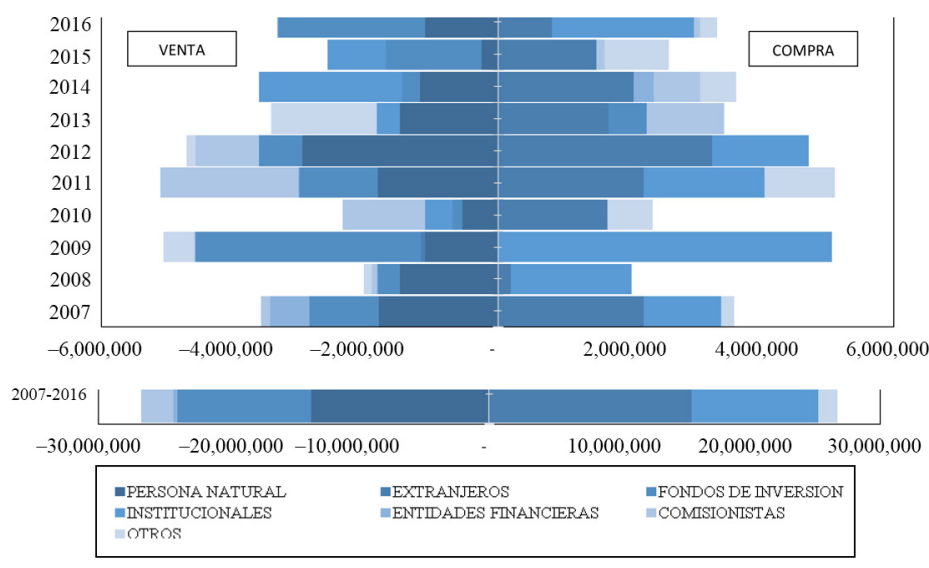

Notas: Se presenta la evolución de la composición del valor transado total en millones de pesos colombianos por agentes del mercado tanto de compra como de venta en la BVC, datos anuales desde 2007 hasta 2016. Elaboración propia

participación sobre el valor transado en el mercado accionario, en comparación con el resto de los agentes.

En contraste, la Tabla II muestra que para el caso colombiano las personas naturales negociaron montos totales por encima de los demás agentes, seguidos por las comisionistas, los fondos de inversión y los institucionales. De hecho, la Figura 3 describe que esto fue así para todos los años hasta 2012 inclusive. Adicionalmente, la Tabla II resalta que los extranjeros muestran una mayor variabilidad en el valor transado, tanto de compra como de venta, presumiblemente porque sus inversiones de portafolio están mayormente expuestas a una dinámica global, altamente incierta[29].

La Figura 6 detalla que las entidades financieras y los agentes institucionales tienen mayores valores promedios por operación que los otros tipos de agentes, debido a las mayores posiciones de los portafolios que gestionan para terceros. Cabe resaltar que las

\begin{tabular}{lrcrr}
\hline & \multicolumn{2}{c}{ Compra } & \multicolumn{2}{c}{ Venta } \\
Tipo de inversionistas & Media & Desv. estándar & Media & Desv. estándar \\
\hline COMISIONISTAS & 523,750 & 280,218 & 503,119 & 263,072 \\
ENTIDADES FINANCIERAS & 6,255 & 32,561 & 3,595 & 28,645 \\
EXTRANJEROS & 386,647 & 336,690 & 517,238 & 368,379 \\
FONDOS DE INVERSIÓN & 651,946 & 334,860 & 566,203 & 298,996 \\
INSTITUCIONALES & 338,053 & 178,467 & 419,704 & 212,219 \\
OTROS & 124,922 & 91,267 & 136,947 & 98,082 \\
PERSONA NATURAL & 897,212 & 401,751 & 782,039 & 376,374 \\
Total & 419,390 & 389,451 & 420,912 & 360,329
\end{tabular}

Notas: La Tabla II presenta la estadística descriptiva del valor transado en millones de pesos colombianos tanto de compra como de venta por tipo de inversionista. Elaboración propia 


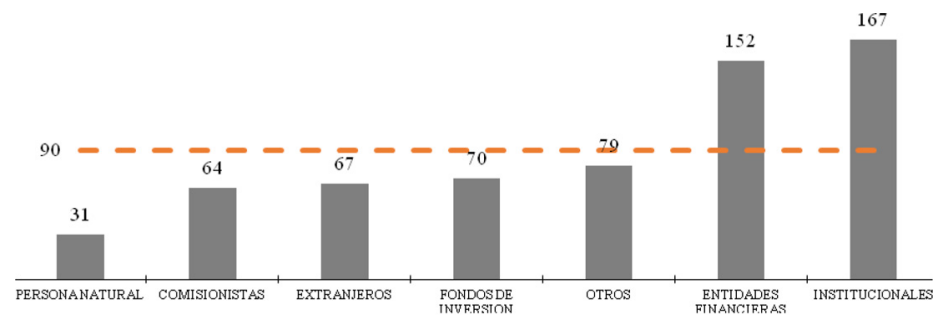

Notas: Se presenta el valor transado en millones de pesos colombianos promedio anual por operación, para los diferentes tipos de agentes en la BVC desde 2007 hasta 2016. Elaboración propia
Determinantes y pronóstico de la actividad bursátil 15 Figura 6. Valor transado promedio por operación

personas naturales, como grupo, negocian montos promedio por operación mucho menores al del resto de los agentes, lo que es de esperarse por sus portafolios de menor monto, pero con un alto número de operaciones.

\subsection{Componente ciclico y tendencial de la actividad bursátil}

La Figura 7 presenta la evolución del valor transado mensual en acciones de la Bvc en el período de estudio. Para facilitar su análisis se aíslan las fluctuaciones de corto plazo mediante el filtro de Hodrick Prescott[30]. Estimando el componente tendencial con dicho procedimiento se evidencian dos períodos importantes marcados por un período alcista hasta finales de 2012 y uno bajista que parece empezar luego del episodio de Interbolsa, que se discute en el numeral 3.1. Por otro lado, no se aprecian comportamientos estacionales claros en el valor transado, aunque esto será puesto a prueba formalmente en el modelo econométrico en el numeral 5.2.

\subsection{Efectos de las emisiones en la actividad bursátil}

Desde 2007 hasta 2016 se realizaron 30 emisiones de acciones en la BVc. Los años con mayor número de emisiones fueron 2007 con nueve, 2011 y 2012 con seis cada uno. En el Anexo B se detallan las emisiones y su respectivo monto de colocación. En la Figura 8, se observa el

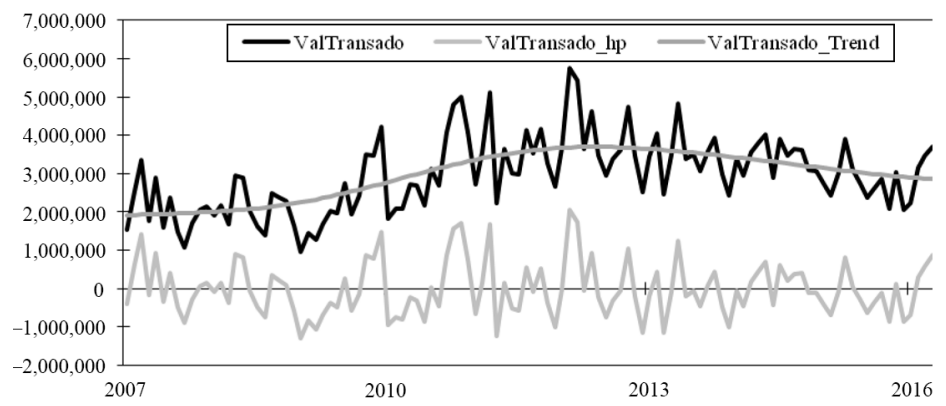

Notas: Se representa el valor transado en millones de pesos colombianos (ValTransado) descompuesto en su componente cíclico (ValTransado hp) y tendencial (ValTransado_Trend), utilizando el filtro de Hodrick Prescott. Elaboración propia

Figura 7. Componente cíclico y tendencial del valor transado 


\section{JEFAS \\ 23,44}

16

Figura 8.

Valor transado y emisiones en el mercado colombiano

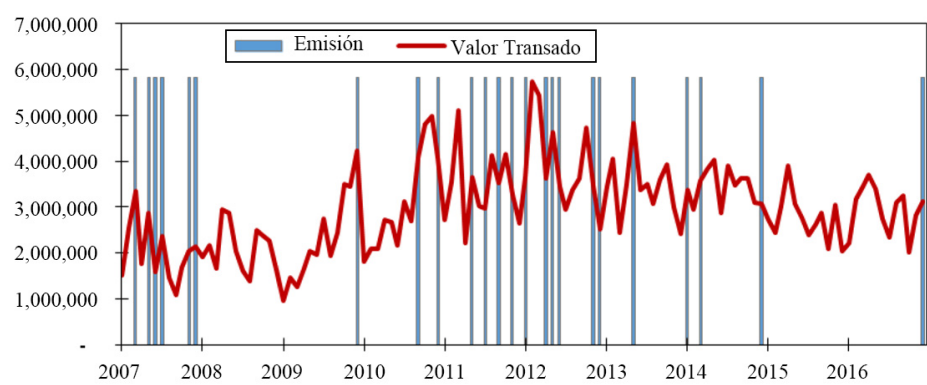

Notas: Se ilustra la evolución del valor transado mensual en millones de pesos colombianos y los meses en que se realizó emisión de acciones en la BVC desde 2007 hasta 2016

Fuente: BVC (2017)

comportamiento mensual del valor transado con relación a los meses de emisiones. Dicha figura sugiere que en los meses donde se realiza la emisión, tiende a aumentar el valor transado. Como se discute en la sección 3 de este trabajo, las emisiones públicas son eventos que capturan el interés del público general, atrayendo al mercado un mayor flujo de agentes no informados, y ofreciéndoles la oportunidad a los institucionales y agentes informados de recomponer sus portafolios.

\section{Resultados empíricos}

Sobre la base de la información recolectada y dadas las características particulares del mercado bursátil colombiano, se plantean dos modelos econométricos sobre la actividad bursátil medida como valor transado. El primero explica su tasa de crecimiento en términos de los cambios porcentuales en el número de operaciones de los tipos de inversionistas más relevantes. El segundo, propone un modelo de pronóstico para la actividad bursátil, explicándola por medio de variables exógenas macro y de sus propios rezagos.

\subsection{Estimación de la tasa de crecimiento de la actividad bursátil}

Es de esperarse que diversos tipos de inversionistas presenten diferentes patrones de compras y ventas según las características de sus portafolios, sus objetivos de inversión y su propio perfil de riesgo. Esta es la motivación para plantear un modelo que capture su impacto en la actividad bursátil agregada. De esta forma, los reguladores y la Bolsa de valores pueden utilizar los resultados para la toma de políticas diferenciadas por agente, en pro del desarrollo del mercado accionario.

La variable dependiente en el modelo será la primera diferencia del logaritmo natural del valor transado, claramente interpretable como la tasa de crecimiento continua de la actividad bursátil, y las variables independientes serán las tasas de crecimiento del número de operaciones de los distintos tipos de inversionistas. En la Tabla III se presentan estadísticas de crecimiento de actividad bursátil por número de operaciones para los tipos de inversionistas referidos para el período de muestra. Conforme a lo descrito en la sección 2 de este estudio, se ve una creciente participación de los extranjeros con una tasa mensual de 2.2 per cent, en contraste con las personas naturales que reducen sus operaciones en promedio en -0.6 per cent mensual. 


\begin{tabular}{|c|c|c|c|c|c|c|}
\hline Variable & $\mathrm{N}$ & Media (\%) & Desv. Estándar (\%) & Minimum (\%) & Maximum (\%) & terminant \\
\hline $\begin{array}{l}\text { Tasa crecimiento ValTransado } \\
\text { Tasa crecimiento Noper }\end{array}$ & 119 & 0.6 & 30.0 & -83.9 & 59.3 & de la actividad \\
\hline $\begin{array}{l}\text { Comisionistas } \\
\text { Tasa crecimiento Noper }\end{array}$ & 118 & 1.0 & 28.2 & -100.2 & 65.2 & at \\
\hline $\begin{array}{l}\text { Extranjeros } \\
\text { Tasa crecimiento Noper Fondos de }\end{array}$ & 118 & 2.2 & 32.0 & -89.1 & 110.2 & \\
\hline $\begin{array}{l}\text { 1 asa crecimiento Noper Fondos de } \\
\text { inversión } \\
\text { Tasa crecimiento Noper }\end{array}$ & 118 & -0.2 & 26.1 & -64.7 & 64.1 & \\
\hline $\begin{array}{l}\text { Institucionales } \\
\text { Tasa crecimiento Noper Personas }\end{array}$ & 118 & 0.7 & 31.6 & -69.3 & 107.3 & \\
\hline naturales & 118 & -0.6 & 25.3 & -75.1 & 71.3 & Tabla III. \\
\hline \multicolumn{7}{|c|}{$\begin{array}{l}\text { Notas: Se presentan las estadísticas descriptivas de la tasa de crecimiento de la actividad bursátil } \\
\text { (ValTransado) en Colombia y las tasas de crecimiento del número de operaciones (Noper) de compra de los } \\
\text { principales tipos de inversionistas, datos mensuales de la Bvc en el período 2007-2016. Elaboración propia }\end{array}$} \\
\hline
\end{tabular}

Un requisito para la modelación con series de tiempo es comprobar si están afectadas por persistentes innovaciones en el proceso generador de los datos. Para resolver este problema, o por lo menos comprender sus posibles efectos, es necesario poner a prueba si las series son estacionarias. Para ello, se utilizan pruebas de raíz unitaria sobre las variables a utilizar en el modelo, tanto en la variable dependiente como en las independientes, como se reporta en la Tabla IV. En todos los casos se rechaza la existencia de raíces unitarias[31].

Al estimar un modelo ARIMA-ARCH de la tasa de crecimiento de la actividad bursátil (Tabla V), se encuentra que la tasa de crecimiento de las operaciones de comisionistas y extranjeros no son estadísticamente significativas para explicar el efecto del crecimiento del valor transado. En contraste, se obtienen efectos estadísticamente significativos y de signo positivo de la tasa de crecimiento de los fondos de inversión, los institucionales y las personas naturales. Es decir, un aumento en la tasa de crecimiento del número de operaciones de dichos agentes en el mercado bursátil colombiano se relaciona de manera más directa con el crecimiento en el valor transado. Todo ello está en concordancia con el análisis previo que los identifica como los agentes más relevantes dentro del grupo de análisis. Para todos los tipos de inversionistas se obtienen

\begin{tabular}{lcc}
\hline & \multicolumn{2}{c}{ Prueba de raíz unitaria } \\
Variable & Dickey-Fuller Aumentado & Phillips-Perron \\
\hline ln_ValTransado & 0.0000 & 0.0000 \\
Tasa crecimiento Noper Comisionistas & 0.0000 & 0.0000 \\
Tasa crecimiento Noper Extranjeros & 0.0000 & 0.0000 \\
Tasa crecimiento Noper Fondos de Inversión & 0.0000 & 0.0000 \\
Tasa crecimiento Noper Institucionales & 0.0000 & 0.0000 \\
Tasa crecimiento Noper Personas Naturales & 0.0000 & 0.0000 \\
VIX & 0.0091 & 0.0116 \\
R_COLCAP & 0.0000 & 0.0000
\end{tabular}

Notas: Se reportan los resultados de las pruebas de raíz unitaria mediante la metodología de Dickey-Fuller y Phillips-Perron para comprobar la estacionariedad del proceso generador de los datos de las series de tiempo utilizadas. Se reporta el $P$-value para cada estadístico. Elaboración propia

Tabla IV. Raíz unitaria 
Tasa crecimiento Noper Comisionistas

Tasa crecimiento Noper Extranjeros

Tasa crecimiento Noper Fondos de inversión

\section{8}

Tasa crecimiento Noper Institucionales
Tasa crecimiento Noper Personas naturales

_cons

AR (1)

AR (2)

MA (3)

MA (4)

$\mathrm{ARCH}(1)$

_cons

$\bar{N}$

Adj $R$-squared

Tabla V.

Prob $>$ F
Tasa crecimiento ValTransado
Resultados estimados de la actividad bursátil por tipo de agente
Notas: Esta tabla reporta los resultados mediante las estimaciones ARCH y ARIMA para la actividad bursátil del mercado colombiano, en el período 2007-2016. La variable dependiente (tasa de crecimiento del ValTransado) hace referencia a la tasa de crecimiento del valor transado total en cada t. Los $p$-values están en paréntesis. * *** $* * *$ representan significancia al 10, 5 y 1\%, respectivamente. Elaboración propia

coeficientes positivos en sus tasas de crecimiento de operaciones, y estadísticamente significativos, como es apenas natural. Ahora bien, el mayor efecto corresponde al número de operaciones de personas naturales, hecho que contrasta con los resultados obtenidos por Chordia et al. (2011). Estos autores encuentran que los inversionistas institucionales y no los individuos, son los responsables de la mayor parte del volumen transado en Estados Unidos, lo que ha llevado a mejoras en la eficiencia de sus mercados accionarios. El resultado para el caso colombiano puede estar explicado por su bajo grado de desarrollo. De hecho, una alta participación de individuos también se ha reportado en otros países emergentes como Corea y Taiwán (Choe et al., 2005; Barber et al., 2009).

Para representar el efecto de las variables y su ajuste a la serie original, se escala la variable con los efectos obtenidos para graficar tanto la variable dependiente real como la variable dependiente estimada. Los resultados se presentan en la Figura 9, que muestra que el comportamiento del valor transado estimado en el modelo se aproxima al comportamiento del valor transado real.

\subsection{Estimación de la actividad bursátil y pronóstico}

Para explicar el comportamiento de la actividad bursátil del mercado accionario colombiano e implementar su pronóstico, se plantea un modelo econométrico que la explique con base en sus propiedades de series de tiempo y variables exógenas explicativas. Para comenzar, la Tabla VI presenta las estadísticas descriptivas del valor transado mensual (ValTransado) de la Bvc entre enero de 2007 y diciembre de 2016, y sus variables explicativas.

Utilizando como variable dependiente el logaritmo natural del valor transado, se estima un modelo ARMA con variables explicativas $\left(X_{t}\right)$. Después de una exploración de la literatura y ensayando diferentes variables económicas y formas alternativas de medirlas, se 


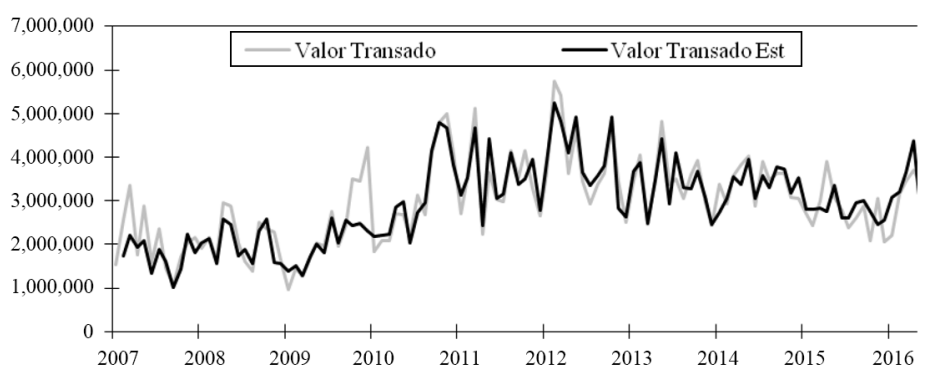

Notas: Se representan tanto el valor transado en millones de pesos colombianos efectivo desde enero de 2007 hasta noviembre de 2016, como el valor estimado por el modelo de tasa de crecimiento de actividad bursátil basado en la actividad de diferentes tipos de agentes (Tabla V) en el mismo período. Elaboración propia con datos de la BVC (2017)
Determinantes y pronóstico de la actividad bursátil

Figura 9. Actividad bursátil estimada sobre la base de la actividad de diferentes tipos de agentes

\begin{tabular}{lccccc}
\hline Variable & $N$ & Media & Desv. estándar & Mínimum & Máximum \\
\hline ValTransado & 120 & $2,949,591$ & 943,593 & 963,096 & $5,741,308$ \\
NumOper & 119 & 54,152 & 14,788 & 25,635 & 100,061 \\
VIX & 120 & 21 & 9 & 10 & 63 \\
r_COLCAP & 119 & 0.23 & 5.08 & -22.40 & 10.45 \\
i & 120 & 5.6 & 2.3 & 3.0 & 10.0
\end{tabular}

Notas: Esta tabla muestra la estadística descriptiva de las variables seleccionadas en el estudio, en el período 2007-2016 con una periodicidad mensual. Elaboración propia con datos de Bloomberg BVC (2017)

Tabla VI.

Estadística descriptiva de las variables de la muestra

encontraron los siguientes tres determinantes estadísticamente significativos de la actividad bursátil en el mercado de acciones de BVC[32]:

- Persistencia: variable dummy que toma el valor de "1" cuando cada uno de los rendimientos pasados de los últimos 3 períodos del índice Colcap son positivos, "0" en cualquier otro caso.

- Emisión: variable dummy, que toma el valor de "1" cuando presenta al menos una emisión en el período; "0" en el caso contrario.

- VIX ${ }_{t-1}$ : nivel del índice VIX en el mes anterior.

A priori se espera que la variable Persistencia tenga un efecto positivo sobre la actividad bursátil, de manera similar al hallazgo de Griffin et al. (2006), quienes reportan que la actividad bursátil presenta una correlación positiva con los rendimientos semanales pasados, particularmente distintiva de mercados poco desarrollados. Como se explicó en la sección 3, desde los modelos de microestructura de mercados se puede inferir que rendimientos positivos (negativos) pasados pueden atraer (ahuyentar) a inversionistas no informados, por ejemplo poco sofisticados y extrapolativos, que asumen una continuación de este comportamiento. De otro lado, también se puede explicar desde el efecto disposición (disposition effect) de Shefrin y Statman (1985) que implica que los inversionistas son más proclives a liquidar posiciones ganadoras, y tienen cierta resistencia a liquidar posiciones vendedoras. 
JEFAS 23,44
Se infiere que las emisiones tengan un efecto positivo sobre la actividad bursátil. Esto puede explicarse en el sentido de Bayraktar (2014), quien muestra que mercados accionarios con mayor número de emisores, tiene mayor actividad bursátil. De otro lado, también es consistente con un mayor número de personas naturales ingresando al mercado por primera vez. Como se explica en la sección 3, los modelos de microestructura de mercados señalan que la actividad bursátil depende en parte del flujo de inversionistas no informados, que parece aumentar en los períodos de emisiones primarias. Además, estas emisiones llevan a los gestores de portafolio a vender otras acciones para balancear sus posiciones.

Por otro lado, se espera que el vix del mes anterior tenga un efecto negativo sobre la actividad bursátil, consistente con el estudio de Chordia et al. (2001), quienes reportan que los aumentos de la volatilidad en el mercado accionario norteamericano tenía efectos negativos sobre la actividad bursátil, tanto de acciones individuales como del agregado del mercado. En los modelos básicos de portafolio, un aumento en la volatilidad percibida de las acciones lleva a los agentes a vender sus posiciones para invertir a la tasa libre de riesgo (Agudelo, 2014), lo que parece reducir el acervo de inversionistas en el mercado en un futuro cercano. Además, es interesante notar que, en versiones alternativas del modelo, este efecto no es significativo para medidas de volatilidad del índice accionario local. Esto parece sugerir que el efecto internacional de desplazamiento hacia inversiones más seguras en tiempos de mayor incertidumbre domina un posible efecto local análogo.

Los resultados del modelo presentados en las dos primeras columnas de la Tabla VII para el valor transado confirman los efectos planteados en las hipótesis. Específicamente emisiones contemporáneas y rendimientos de meses pasados tienen un efecto positivo sobre la actividad bursátil. Este resultado es acorde a lo evidenciado por Bayraktar (2014). Adicionalmente, se confirma el efecto que denominamos "persistencia"; es decir, rendimientos positivos de cada uno de los últimos tres meses del COLCAP se asocian con aumento en el valor transado, efecto cualitativamente similar al reportado por Griffin et al. (2006). Además, se evidencia que el rezago de la volatilidad (vix) tiene un efecto negativo sobre la actividad bursátil del mercado accionario colombiano, lo que pone de manifiesto la importancia de la dinámica bursátil en Estados Unidos para el mercado de valores colombiano.

La Tabla VII también presenta los resultados de emplear, como medidas alternativas de actividad bursátil, el número total de operaciones y la rotación ("turnover"). En el caso del número de operaciones, el modelo (3) con variables exógenas ratifica tanto el efecto positivo de la "persistencia", como el negativo del vix, reportados para el valor transado. Caso contrario, el efecto de la variable "emisión" es positivo, pero no es estadísticamente significativo. Por su parte, la quinta columna presenta la modelación de la rotación (turnover) del mercado con variables exógenas. Dichos resultados confirman el efecto positivo de las emisiones, reportado para el valor transado así como el de la variable "persistencia". Se presenta también el efecto negativo del vix como en las columnas (1) y (3), pero sin significancia estadística. En conclusión, podemos afirmar que las medidas alternativas de actividad bursátil confirman en gran parte los hallazgos reportados para la variable principal de este estudio, el valor transado total en el mercado accionario.

Es importante resaltar la gran capacidad explicativa que tiene el modelo ARIMA por sí solo, capturando un importante comportamiento inercial de la misma serie de tiempo del valor transado. Concretamente, el $R^{2}$ ajustado que se reporta en la Tabla VII revela que el 47 per cent del proceso generador de los datos en la modelación del valor transado está sujeto al comportamiento de los componentes AR y MA del modelo propuesto. Es decir, el comportamiento pasado del valor transado en el mes anterior y las innovaciones de 5 y 12 meses atrás explican en gran proporción el comportamiento actual de la misma variable. 


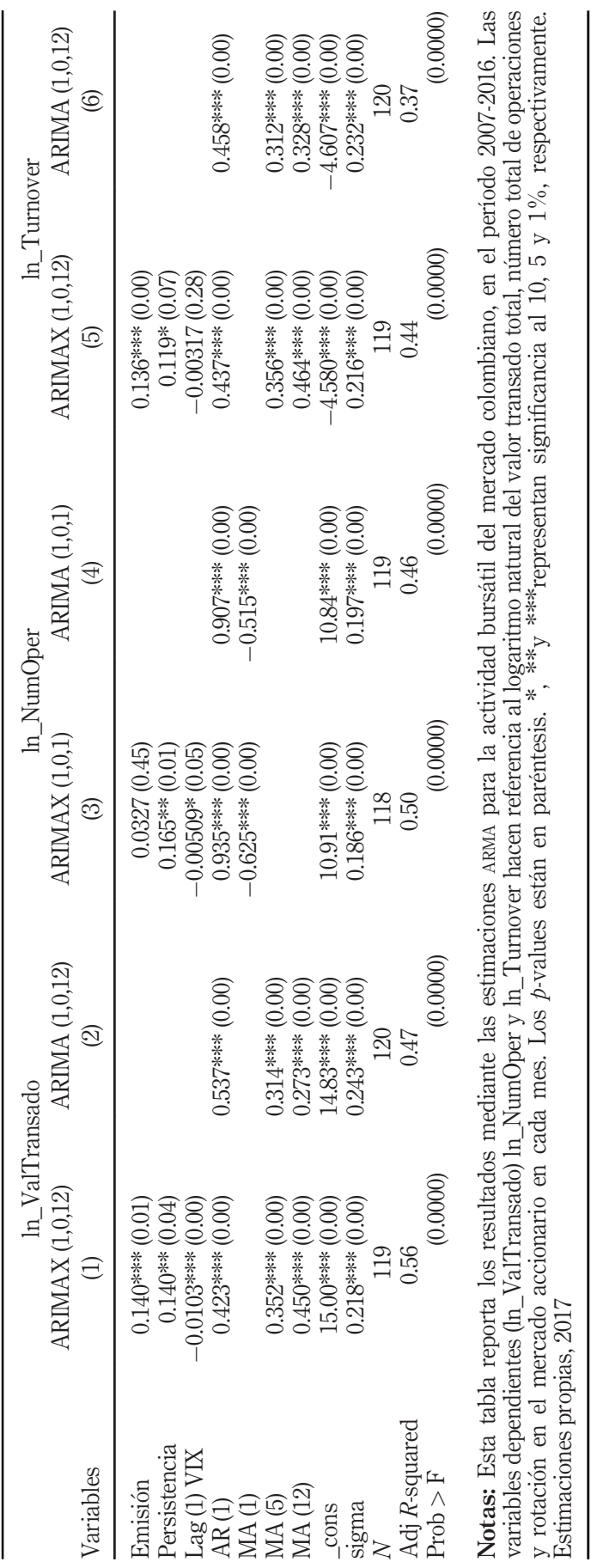

Determinantes y pronóstico de la actividad bursátil

21

Tabla VII.

Resultados estimados de la actividad bursátil 
JEFAS

23,44

Para los modelos de número de operaciones y rotación estas cifras son de 46 y 37 per cent, respectivamente.

En la Figura 10 se representa la evolución de la variable de valor transado estimada mediante el modelo, junto con los valores que efectivamente presentó. Se observa que el modelo estimado sigue un comportamiento similar al valor transado real.

\section{Conclusiones}

El estudio de la actividad bursátil en el mercado accionario colombiano entre enero de 2007 a diciembre de 2016, permite determinar su evolución en el tiempo, su dependencia temporal y su relación con variables externas de mercado. De este estudio se desprenden las siguientes conclusiones principales.

En primer lugar, existen diferencias significativas en el promedio del valor transado de los tipos inversionistas. Si bien no sorprende que la mayor parte de la actividad bursátil proceda de instituciones, es algo más inesperado que las personas naturales sean responsables de una parte importante. A su vez, los extranjeros han aumentado su participación, específicamente como compradores netos; sin embargo, su participación es la más volátil, y parece depender de la coyuntura económica global.

Por otro lado, se observa la importancia de los efectos autorregresivos y de media móvil de la actividad bursátil, que manifiesta una significativa predictibilidad en esta variable de mercado. En particular, el valor transado del mes actual está explicado en parte por el volumen transado del mes anterior y efectos de cinco y 12 meses. Estos dos últimos parecen capturar efectos estacionales dinámicos. Con estas solas tres variables autorregresivas, el modelo econométrico puede explicar un 47 per cent de la variación de la actividad bursátil mes a mes. Tentativamente, la estacionalidad de 12 meses puede explicarse por ciclos anuales de liquidez en la economía. El efecto de un mes puede explicarse por la mayor atención por parte de agentes no informados a un mercado accionario particularmente activo en el pasado reciente. Futuros estudios podrían indagar por los determinantes de estas regularidades.

En tercer lugar, la actividad bursátil tiene una relación positiva con las emisiones de acciones en el mismo mes. Aunque este tipo de eventos no tiene un efecto directo en meses subsiguientes, sí lo tiene de manera indirecta a través del efecto del valor transado de los

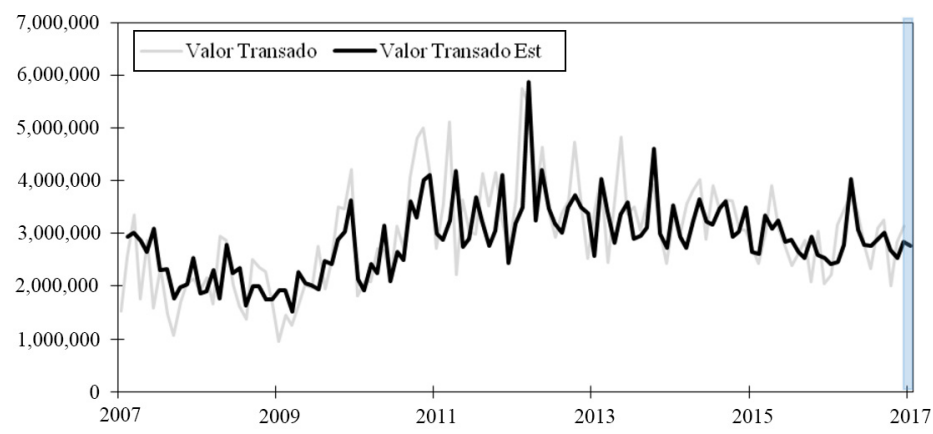

Figura 10.

Actividad bursátil estimada sobre la base de variables exógenas
Notas: Se representan tanto el valor transado en millones de pesos colombianos efectivo desde enero de 2007 hasta noviembre de 2016, como el valor estimado por el modelo basado en un modelo ARIMA con variables exógenas (Columna 1, Tabla VII) en el mismo período. Elaboración propia con datos de la BVC (2017) 
meses anteriores mencionados en el párrafo anterior. Claramente, las emisiones atraen a un número importante de agentes no informados al mercado accionario, y brindan la oportunidad a otros agentes de liquidar posiciones importantes. Se deja para posteriores estudios explorar la dinámica de los efectos de las emisiones en la actividad bursátil y liquidez de todo el mercado, entendiendo que seguramente su efecto no se inscribe solamente a la acción emitida sino que beneficia a buena parte del mercado.

Por otra parte, se observa el efecto de la persistencia de los rendimientos pasados positivos en la actividad bursátil, similar a lo reportado en la literatura. Es decir, meses de rendimientos positivos (negativos) inducen una mayor (menor) transacción de títulos valores en el mercado accionario colombiano. Este efecto asimétrico ya ha sido reportado en otros mercados y otros períodos en la literatura, y es más fuerte en mercados menos sofisticados y eficientes. Futuros estudios podrían explorar si este efecto puede explicarse por un sesgo de atención de los agentes no informados de mercados accionarios alcistas en el pasado reciente.

Finalmente, la volatilidad en los mercados financieros internacionales repercute de manera negativa en la actividad bursátil local, coherente con hallazgos previos de la literatura. Esto es consistente con un efecto de "fuga a la calidad", según el cual cuando los inversionistas perciben mayor riesgo sobre las acciones, desplazan sus posiciones a activos refugios. Se resalta el hecho de que dicho efecto es de índole global, más determinado por la expectativa de la volatilidad del SP500, medida por el VIX, que por la volatilidad contemporánea del COLCAP. Con los datos detallados disponibles para el mercado accionario de Colombia, con operaciones por diferentes tipos de agentes, se puede explorar en otras investigaciones cuáles son los tipos de agentes que más inducen este efecto de fuga a la calidad tanto en la actividad bursátil como en los precios.

\section{Notas}

1. Los dos conceptos son empleados de manera indistinta en la prensa y por los practitioners, pero en los estudios académicos hay una clara distinción.

2. Adicionalmente, el presente estudio se diferencia de Agudelo (2009), en el período de estudio (1997-2007 versus 2007-2016), la frecuencia (diaria versus mensual), la intención (inferencia versus modelo de pronóstico), modelo econométrico y variables específicas en el mismo. Además, la pregunta central de dicho artículo es establecer el efecto sobre la actividad bursátil de la fusión de las tres Bolsas existentes en Colombia hasta julio de 2001, y que a partir de dicho mes pasaron a conformar la Bolsa de Valores de Colombia (BVC, 2009).

3. Estadísticas de la wFE (World Federation Exchanges).

4. Según la Superintendencia Financiera de Colombia (2008) los emisores de valores son entidades de carácter público o privado, que ponen en circulación títulos valores, bien sean representativos de deuda, de propiedad, de tradición o de participación, que deben inscribirse en el Registro Nacional de Valores y Emisores.

5. Veinticinco son las acciones pertenecientes actualmente al índice colCAP, el principal de la Bolsa. La Superintendencia Financiera de Colombia realiza la clasificación de bursatilidad de las acciones cada mes con reclasificación trimestral, según la definición de una función de liquidez que depende del volumen, la rotación y la frecuencia con la que se transe una acción. (Manual Básico de Operación del Nuevo Modelo de Acciones, BVC, 2010a, 2010b).

6. Informe de gestión BVC (2007).

7. También se destacan la aprobación de la ley de sostenibilidad fiscal y regalías (reforma constitucional), la promoción del plan económico para el cuatrienio y se expidió la reglamentación que permite la negociación de valores de renta variable en el Mercado Integrado Latinoamericano (MLA). 
8. Los ocho emisores fueron: Avianca-Taca, Grupo Aval, Nutresa, Almacenes Éxito, Ecopetrol, Empresa de Energía de Bogotá, Banco Davivienda y Grupo de Inversiones Suramericana.

9. Informe de gestión de la BVC (2011).

10. Los problemas de liquidez de la comisionista están asociados tanto a operaciones altamente riesgosas como a manejos irregulares. En particular, los problemas surgieron por las operaciones repo en la acción de Fabricato, la misma Interbolsa y otras de limitada liquidez. Al parecer estas operaciones comenzaron desde 2011, año en el que la acción de Fabricato se apreció cerca de un 200\%, a pesar de una desvalorización del 16\% del IGBC. Esto ha sido explicado por la manipulación a través de repos y transacciones preacordadas por parte de Interbolsa o agentes asociados. La intervención de Interbolsa se traduce necesariamente en una disminución de la actividad bursátil, ya que esta entidad tenía cerca del 34\% de participación en el mercado Bursátil de Colombia (Informe de Gestión BVC, 2012). Más aún, se ve afectada la confianza y credibilidad de los inversionistas en el mercado bursátil en general, sobre todo de las personas naturales.

11. Informe de Gestión de la BVC (2015a, 2015b).

12. Informe de Gestión de la BVC (2016).

13. Estudio sobre datos diarios del índice accionario NYSE (The New York Stock Exchange) y el S\&P500 (Standard and Poor's) entre 1928 y 1987, mediante una estimación Semi No Paramétrica (SNP).

14. Miden la actividad bursátil mediante el volumen y el número de operaciones. La liquidez mediante el quoted spread (el diferencial de cotización de la transacción del bid-ask), effective spread (la diferencia entre el precio de ejecución y el punto medio de la cotización del bid-ask vigente) y depth (el promedio de las profundidades de oferta y demanda cotizadas).

15. Datos del índice NYsE entre 1988-1998 con periodicidad diaria.

16. El desbalance de órdenes se mide como la diferencia entre las compras activas y las ventas activas (ya sea por monto, número de operaciones o número de acciones). Se entiende como compra (venta) activa, aquella transacción iniciada por el comprador (vendedor), mediante una orden de mercado $\mathrm{u}$ orden límite inmediatamente ejecutable $\mathrm{y}$, por ende, con un vendedor (comprador) que estaba suministrando liquidez mediante una orden límite de venta (compra) existente. La actividad bursátil mediante el volumen y el número de operaciones. La liquidez mediante el bid-ask spread.

17. Datos diarios de las acciones del NYSE entre 1988-1998 y como índice de referencia del mercado el S\&P500.

18. En otro estudio relacionado, Chordia et al. (2005), presentan evidencia de una interacción de la liquidez del mercado accionario y del mercado de renta fija por medio de la actividad bursátil, debido a las estrategias de asset allocation de los portafolios de los inversionistas. Choques negativos en los mercados bursátiles pueden inducir episodios de flight to quality, es decir, migración de posiciones en acciones a bonos, o movimientos complementarios. Este estudio emplea datos diarios entre 1991-1998 de acciones del NYSE y los bonos del Tesoro de Estados Unidos.

19. Valor transado normalizado por la capitalización del mercado.

20. Datos semanales desde 1993-2003 para 46 países.

21. Datos mensuales desde 1963-2002 de las acciones del NYSE y Nasdaq.

22. Panel de datos de inversores individuales con cuentas en brokers durante un período de 51 meses, además de posiciones de portafolios de 3.079 inversores durante 1997-2001.

23. Datos desde 1993-2008 del NYSE. 
24. Por ejemplo, en julio de 2015 hay acciones entre $\$ 7$ y $\$ 20$, como Tablemac, Fabricato, Enka, BVC, pero también acciones entre $\$ 30,000$ y $\$ 60,000$ como PF Bancolombia, Grupo Sura, Corficol y Banco de Bogotá.

25. Ver, por ejemplo: www.nyse.com/publicdocs/nyse/markets/nyse/NYSE_Price_List.pdf. El esquema general de las comisiones de $B V C$ se discute en el Anexo $\mathrm{A}$.

26. En el sentido amplio o débil significa que el proceso generador de los datos tiene media, varianza y autocorrelaciones constantes.

Determinantes y pronóstico de la actividad bursátil

27. Los modelos ARCH (Autorregresive Conditional Heterocedasticity) fueron propuestos inicialmente por Engle (1982).

28. Se encuentra evidencia del problema de autocorrelación luego de la prueba de Ljung-Box Q para series de tiempo, por medio de la función de autocorrelación y la autocorrelación parcial. Adicionalmente, se toma el estadístico de Durbin-Watson para detectar la presencia de autocorrelación.

29. Se usa el estadístico de prueba robusta de Levene para la igualdad de varianzas entre los grupos definidos y los dos estadísticos propuestos por Brown y Forsythe, que reemplazan la media en la fórmula de Levene con otros estimadores de localización. La primera alternativa reemplaza la media con la mediana. La segunda alternativa reemplaza la media con la media recortada del $10 \%$. Bajo todos los criterios, se rechaza la hipótesis nula de varianza constante para las medias a través del tiempo en el grupo de inversionistas.

30. El filtro de Hodrick-Prescott separa en el componente tendencial del componente cíclico. El componente tendencial puede provenir tanto de una tendencia determinística, como de una estocástica. El parámetro de suavización lambda determina los periodos del ciclo estocástico que siguen el componente cíclico estacionario. La elección de lambda es arbitraria, pero Hodrick y Prescott estiman para datos mensuales un valor para el parámetro de 14400.

31. Las variables que se incrementan a lo largo del tiempo constituyen ejemplos de series no estacionarias. Hay también series que no aumentan a través del tiempo, pero donde los efectos de las innovaciones no se extinguen con el tiempo. Éstas también son no estacionarias. Si los errores estándar producidos son sesgados, implica que el criterio convencional usado para juzgar si existe o no una relación causal entre las variables no es confiable. En muchos casos se descubre una relación significativa cuando en realidad no existe; una regresión donde esto ocurre recibe el calificativo de regresión espuria.

32. En modelos alternativos, disponibles por solicitud a los autores, se consideraron otras variables previamente empleadas por la literatura. Específicamente: la tasa de interés (IBR, DTF y la tasa de intervención del Banco de la República), la capitalización bursátil, la tasa de cambio, crecimientos del IPC y del PIB. También rendimientos de meses individuales pasados, volatilidad mensual del ColCAP, dummies por mes del año, y dummies por posibles efectos permanentes de la crisis de Interbolsa y del $X$-Stream. Todas estas variables se descartaron por insuficiente poder explicativo.

33. Por ejemplo, para 2017 BTG cobra el 3\% de comisión máxima por operación realizada en renta variable y Ultraserfinco cobra un mínimo de 0.5\% y un máximo del 7\% (BTG, 2017; Ultraserfinco, 2017).

\section{Referencias}

Agudelo, D. (2009), Trading Activity in Colombian Stock Markets. Determinants and Evolution 1997-2007, Ad-minister Universidad EAFIT, pp. 89-112.

Agudelo, D. (2014), Inversiones en renta variable, Fondo Editorial Universidad EAFIT.

Agudelo, D., Gutiérrez, A. y Múnera, N. (2014), "Market quality and structural changes in the trading system: the case of X-stream on the colombian stock exchange", Academia Revista Latinoamericana De Administración, Vol. 27 No. 3, pp. 324-330. 
JEFAS 23,44

Allen, F. y Santomero, A.M. (1998), "The theory of financial intermediation”, Journal of Banking \& Finance, pp. 146-1485.

Barber, B.M., Lee, Y.T., Liu, Y.J. y Odean, T. (2009), "Just how much do individual investors lose by trading?", Review of Financial Studies, Vol. 22 No. 2, pp. 609-632.

Bayraktar, N. (2014), "Measuring relative development level of stock markets: capacity and effort of countries", Borsa Istanbul Review, Vol. 14 No. 2, pp. 74-95.

Box, G.E.P. y Jenkins, G.M. (1973), "Some comments on a paper by Chatfield and Prothero and on a review by Kendall", Journal of the Royal Statistical Society. Series A (General), Vol. 136 No. 3, pp. 337-352.

Box, G., Jenkins, G.M. y Reinsel, G.C. (1994), Time Series Analysis: Forecasting and Control, 3rd ed., Prentice-Hall, Englewood.

BTG (2017), Tarifas. Recuperado el 3 de abril de 2017 de, available at: www.btgpactual.com.co/images/ userfiles/files/formas/wm/Politica\%20Tarifas/Tarifas.pdf

BVC (2007), Informe de gestión. Recuperado el 3 de abril de 2017, de: www.bvc.com.co/pps/tibco/ portalbvc/Home/Accionistas

BVC (2009), Reporte Anual 2009. Recuperado el 20 de marzo de 2017, de: www.bvc.com.co/pps/tibco/ portalbvc/Home/Accionistas

BVC (2010a), "Informe de gestión. Recuperado el 3 de abril de 2017", de: www.bvc.com.co/pps/tibco/ portalbvc/Home/Accionistas

BVC (2010b). Manual básico de operación del nuevo modelo de acciones. Recuperado el 13 de enero de 2017, de: www.bvc.com.co/cursos/administracion/editor/homeFiles/Manual_Basico_de_Operacion_del_Nuevo_ Modelo_de_Acciones.pdf

BVC (2011), Informe de gestión. Recuperado el 3 de abril 2017, de: www.bvc.com.co/pps/tibco/ portalbvc/Home/Accionistas

BVC (2012), Informe de gestión. Recuperado el 3 de abril de 2017, de: www.bvc.com.co/pps/tibco/ portalbvc/Home/Accionistas

BVC (2015a). Estados financieros individuales y consolidados 2015. Recuperado el 20 de abril de 2017 de BVC: www.bvc.com.co/pps/tibco/portalbvc/Home/Accionistas

BVC (2015b), Informe de gestión. Recuperado el 3 de abril de 2017, de: www.bvc.com.co/pps/tibco/ portalbvc/Home/Accionistas

BVC (2016), Informe de gestión. Recuperado el 3 de abril de 2017, de: www.bvc.com.co/pps/tibco/ portalbvc/Home/Accionistas

BVC (2017), Tarifas renta variable. Recuperado el 20 de marzo de 2017 de: www.bvc.com.co/pps/tibco/ portalbvc/Home/Accionistas

Choe, H., Kho, B.C. y Stulz, R.M. (2005), "Do domestic investors have an edge? the trading experience of foreign investors in Korea”, Review of Financial Studies, Vol. 18 No. 3, pp. 795-829.

Chordia, T., Huh, S.W. y Subrahmanyam, A. (2006), "The cross-section of expected trading activity", Review of Financial Studies, Vol. 20 No. 3, pp. 709-740.

Chordia, T., Roll, R. y Subrahmanyam, A. (2001), "Market liquidity and trading activity", The Journal of Finance, Vol. 56 No. 2, pp. 501-530.

Chordia, T., Roll, R. y Subrahmanyam, A. (2002), "Order imbalance, liquidity, and market returns", Journal of Financial Economics, Vol. 65 No. 1, pp. 111-130.

Chordia, T., Roll, R. y Subrahmanyam, A. (2011), "Recent trends intrading activity and market quality", Journal of Financial Economics, Vol. 101 No. 2, pp. 243-263.

Chordia, T., Sarkar, A. y Subrahmanyam, A. (2005), "An empirical analysis of stock and bond market liquidity”, The Review of Financial Studies, pp. 86-129.

Engel, R. (1982), "Autorregresive conditional heterocedasticity with estimates of the variance of the UK inflation”, The Econometric Society, pp. 987-1007. 
Gallant, R., Rossi, P. y Tauchen, G. (1992), "Stock prices and volume”, Review of Financial Studies, Vol. 5 No. 2, pp. 199-242.

Glaser, M. y Weber, M. (2009), “Which past returns affect trading”, Journal of Financial Markets, Vol. 12 No. 1, pp. 1-31.

Glosten, L.R. y Milgrom, P.R. (1985), "Bid, ask and transaction prices in a specialist market with heterogeneously informed traders", Journal of Financial Economics, Vol. 14 No. 1, pp. 71-100.

Griffin, J., Nardari, F. y Stulz, R. (2006), "Do investors trade more when stocks have performed well? Evidence from 46 countries", Review of Financial Studies, pp. 905-951.

Harris, L. (2003), Trading and Exchanges. Market Microstructure for Practitioners, Oxford University Press, New York, NY.

Kyle, A.S. (1985), “Continuous auctions and insider trading”, Econometrica, Vol. 53 No. 6, pp. 1315-1335.

Lo, A.W. y Wang, J. (2000), "Trading volume: definitions, data analysis, and implications of portfolio theory", Review of Financial Studies, Vol. 13 No. 2, pp. 257-300.

Shefrin, H. (2002), Beyond Greed and Fear: Understanding Behavioral Finance and the Psychology of Investing, Oxford University Press on Demand.

Shefrin, H. y Statman, M. (1985), "The disposition to sell winners too early and ride losers too long: theory and evidence", Journal of Finance, Vol. 40 No. 3, pp. 777-790.

Stoll, H.R. (2000), “Presidential address: friction”, Journal of Finance, Vol. 55 No. 4, pp. 1479-1514.

Superintendencia Financiera (2007), Tarifas, comisionistas de bolsa, Recuperado el 15 de noviembre de 2017 de: www.superfinanciera.gov.co/jsp/loader.jsf?1Servicio=Publicaciones\&lTipo= publicaciones\&1Funcion $=$ loadContenidoPublicacion\&id $=15910$

Superintendencia Financiera de Colombia (2008), Conceptos generales del Mercado de Valores, Recuperado el 03 de abril de 2017, de: www.superfinanciera.gov.co/SFCant/ ConsumidorFinanciero/conceptosbasicosmv.pdf

\section{Lecturas sugeridas}

Maginn, J.L., Tuttle, D.L., McLeavey, D.W. y Pinto, J.E. (2007), The Portfolio Management Process and the Investment, CFA Institute, Hoboken, New Jersey.

Ultraserfinco (2017), Tarifas, Recuperado el 20 de marzo de 2017 de: www.ultraserfinco.com/site/ Portals/0/Documentos/Tarifas/Tarifas_Costos_Ultraserfinco_2015.pdf

\section{Anexo A - Comisiones en la $B V C$}

De acuerdo a la Superintendencia Financiera (2007) cada comisionista de bolsa decide la tarifa a cobrar por sus servicios. Naturalmente, ante un mayor número de operaciones y montos transados (actividad bursátil), mayores son los ingresos generados por comisiones cobradas por las scB[33].

Los cargos variables por operación están en función de volumen transado, es decir, a mayor actividad bursátil mayor es la tarifa cobrada a las SCB, a estas se les cobra según el tipo de plan al cual estén afiliados (básico, profesional 1 o 2 y platinum) y de acuerdo al plan se cobra un porcentaje por el valor por volumen transado por punta en el mes. Además, cobran tarifas por la emisión y manutención de acciones; es decir, a mayor número de emisores mayor serán los ingresos por este rubro (Tarifas renta variable bvc, 2017).

Los ingresos de la Bvc relacionados con la actividad bursátil son los asociados a operaciones de renta variable, representando en 2015 un 26.8 per cent de los ingresos por actividades ordinarias, lo que pone de manifiesto la importancia de la actividad bursátil en los ingresos de la BVc. Dichos ingresos disminuyeron un 19.9 per cent con respecto a 2014, debido al menor volumen operado en el mercado de contado ocasionado por la coyuntura del mercado, donde los precios de 
JEFAS 23,44

28

los commodities cayeron. La tarifa promedio implícita por millón disminuyó un 5 per cent, debido a un menor volumen de operaciones realizadas (Estados financieros individuales y consolidados de la Bvc, 2015).

Otro ingreso a resaltar es el asociado a la inscripción y sostenimiento de títulos, que representan un 20.5 per cent de los ingresos ordinarios, los cuales disminuyeron un 5 per cent con respecto a 2014, dado el menor financiamiento de las compañías vía mercados de capitales.

\section{Anexo B - Emisión de acciones en BVC}

\begin{tabular}{lcr}
\hline Emisor & Fecha de colocación & Monto colocado \\
\hline Almacenes Éxito SA & $14 / 03 / 2007$ & 259,350 \\
ISAGEN & $25 / 05 / 2007$ & 488,325 \\
Grupo Aval & $21 / 06 / 2007$ & 202,984 \\
ISAGEN & $27 / 07 / 2007$ & 103,764 \\
Bancolombia SA & $09 / 07 / 2007$ & 323,976 \\
Bolsa Nacional Agropecuaria & $06 / 11 / 2007$ & 20,000 \\
Ecopetrol SA & $27 / 11 / 2007$ & $5,722,813$ \\
Banco de Crédito & $05 / 12 / 2007$ & 282,000 \\
Interconexión Eléctrica SA & $06 / 12 / 2007$ & 399,045 \\
Interconexión Eléctrica SA ESP & $09 / 12 / 2009$ & 384,198 \\
Banco Davivienda SA & $20 / 09 / 2010$ & 419,354 \\
Constructora Conconcreto SA & $20 / 12 / 2010$ & 94,680 \\
Fogansa SA & $30 / 12 / 2010$ & 10,406 \\
AviancaTaca Holding SA & $05 / 05 / 2011$ & 500,000 \\
Grupo Nutresa SA & $14 / 07 / 2011$ & 522,500 \\
Ecopetrol SA & $30 / 09 / 2011$ & $2,383,488$ \\
Empresa de Energía de Bogotá SA ESP & $02 / 11 / 2011$ & 772,227 \\
Banco Davivienda SA & $25 / 11 / 2011$ & 716,193 \\
Grupo de Inversiones Suramericana SA & $28 / 11 / 2011$ & $3,455,886$ \\
Bancolombia SA & $31 / 01 / 2012$ & $1,132,139$ \\
Construcciones El Cóndor SA & $02 / 04 / 2012$ & 162,584 \\
Carvajal Empaques SA & $29 / 05 / 2012$ & 195,752 \\
Acerías Paz del Rí́ SA & $01 / 06 / 2012$ & 271,390 \\
Cemex Latam Holdings SA & $13 / 11 / 2012$ & 278,731 \\
Constructora Conconcreto SA & $26 / 12 / 2012$ & 243,000 \\
Cementos Argos SA & $10 / 05 / 2013$ & $1,401,400$ \\
Grupo Aval Acciones y Valores SA & $22 / 01 / 2014$ & $2,411,730$ \\
Bancolombia SA & $03 / 03 / 2014$ & $2,662,000$ \\
Banco de Bogotá SA & $15 / 12 / 2014$ & $1,500,000$ \\
Grupo Argos SA & $27 / 12 / 2016$ & 676,964 \\
& & \\
& &
\end{tabular}

Tabla AI.

Emisión de acciones en la $B V C$
Nota: La Tabla AI reporta las emisiones de acciones y monto colocado (en millones de pesos) en la $B V C$ desde el 2007 hasta 2016 , fuente $B V C$

\section{Autor para correspondencia}

Diego A. Agudelo puede ser contactado al: dagudelo@eafit.edu.co

For instructions on how to order reprints of this article, please visit our website: 